CHAPITRE 9

\title{
Le cas particulier des accidents graves
}

Jean-Pierre Pervès 
1 Quelles différences entre démantèlement normal et démantèlement post-accidentel ?

2 Quelques rappels d'accidents graves

3 Retours d'expérience des accidents

4 Retours d'expérience des mises en sécurité et des démantèlements après accident

4.1. Three Mile Island (réacteur à eau pressurisée de 900 MW)

4.2. Tchernobyl (réacteur graphite/eau de $1000 \mathrm{MW}$ )

4.3. Fukushima (quatre réacteurs à eau bouillante de 400 à $550 \mathrm{MW}$ )

Conclusion et perspectives 


\section{Quelles différences entre démantèlement normal et démantèlement post-accidentel ?}

Peut-on comparer les opérations de démantèlement d'un îlot de centrale nucléaire normalement programmé, puis arrêté, et le démantèlement $d$ 'une telle installation après un accident grave conduisant à une fusion du cœur ?

Les circonstances sont bien sûr très différentes :

- d'une part, prévision, préparation, évaluation par l'Autorité de sûreté, technologies qualifiées, cycle des déchets maîtrisé, chantier confiné ;

- d'autre part, soudaineté des événements, difficulté à évaluer la situation, inaccessibilité, mesures d'urgence de protection du personnel et de la population, moyens techniques inadaptés, et étendue du territoire à gérer.

L'objectif d'une telle comparaison serait de rapprocher autant que faire se peut les moyens d'action dans ces deux situations si différentes, en s'appuyant sur les technologies développées pour les démantèlements programmés en fin d'exploitation, qui, elles, ont l'avantage d'être opérationnelles et qualifiées'.

Cependant, les situations sont si différentes qu'il faut avoir recours à de nouvelles technologies. Par exemple :

- la dosimétrie sera beaucoup plus forte, les accès seront difficiles et de nouvelles techniques, largement télé-opérées et durcies aux rayonnements sont à développer ;

- les thématiques déchets seront également très différentes compte tenu de la présence d'un corium (mélange de cœur fondu et de matériaux divers) pour lequel une filière de traitement, entreposage et stockage devra être créée, et de quantités très importantes de déchets ${ }^{2}$ radioactifs solides et effluents radioactifs liquides contaminés et parfois très irradiants ;

- en cas de contamination hors de la centrale, les autorités sont confrontées à la nécessité de décontaminer des lieux de vie et des sols sur de très grandes surfaces, situation complètement inconnue dans une hypothèse de démantèlement classique.

De manière générale, dans une situation classique de démantèlement de réacteur, le cœur de celui-ci, qui contient la quasi-totalité de la radioactivité,

\footnotetext{
1 Guidez J. et al. (2013). Les défis du démantèlement, une réalité industrielle, des perspectives. Revue générale nucléaire $\mathrm{n}^{\circ} 4$.

${ }^{2}$ Les déchets d'un réacteur de centrale électronucléaire accidenté gravement contiennent en particulier des actinides (les isotopes du plutonium, de l'américium, du curium), des produits de fission (avec leurs descendants par désintégrations $\beta, \gamma$ ) et du tritium.
} 
a été évacué, le volume irradiant étant limité à la cuve et sa proximité. Dans une situation accidentelle, une part qui peut être importante de l'inventaire radiologique du cœur est dispersée dans un volume plus ou moins grand (circuit primaire, enceinte de confinement et éventuellement environnement).

\section{Quelques rappels d'accidents graves}

Une installation, hors réacteurs, a été accidentée avec un impact important sur l'environnement à Mayak ${ }^{3}$ en URSS en 1957 (explosion chimique d'une cuve de résidus du traitement de combustibles usés, contamination élevée $d^{\prime}$ 'un lac et $d$ 'une rivière et injection de liquides très radioactifs en profondeur), et a nécessité une évacuation durable des populations voisines.

Mais les trois accidents qui ont le plus marqué ont été Three Mile Island (TMI) aux États-Unis en 1979, Tchernobyl en URSS en 1986, et Fukushima au Japon en $2011^{4}$. Suite à ces accidents, les règles de conception, construction et exploitation des installations nucléaires, et des réacteurs en particulier, ont été profondément remaniées pour renforcer leur résistance et, parallèlement, les autorités de sûreté ont durci la réglementation et édicté de nouvelles

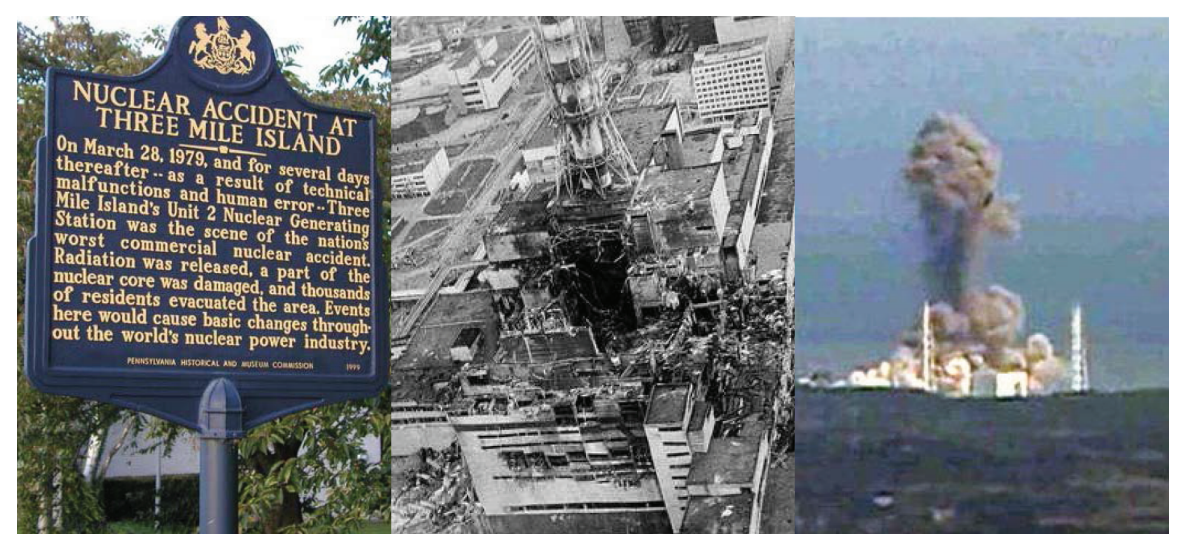

Figure 9.1

TMI, la centrale de Tchernobyl et une explosion H2 à Fukushima.

3 Voir : Kyshtym desaster http://www.iaea.org/inis/collection/NCLCollectionStore/_Public/14/724059.pdf. Mayak est le nom du lieu-dit, situé près de la ville de Ozyorsk, Tchélyabinsk Oblast, le site de production de plutonium étant désigné par l'usine Mayak. Celle-ci est aussi désignée par Tchélyabinsk-40 ou Tchélyabinsk-65.

${ }^{4}$ L'accident de Saint-Laurent-des-Eaux en 1969, classé au niveau 4 de l'échelle INES, n'a pas eu de conséquences notables sur l'environnement. L'accident de niveau 5 du réacteur graphite-gaz de Windscale en 1957, avec incendie du graphite, a eu des conséquences externes limitées. 
normes. La prise en compte d'accidents graves dans les réacteurs, afin d'en limiter les conséquences, résulte d'une analyse du retour d'expérience de ces accidents du passé, très différents les uns des autres.

\section{Retours d'expérience des accidents}

Ces accidents ont eu des origines très diverses, sur des réacteurs de puissance de conceptions assez radicalement différentes et ils ont eu des conséquences très inégales: on peut cependant en tirer des enseignements souvent très complémentaires, des retours d'expérience (REX), qui ont été analysés en grand détail en France par l'Autorité de sûreté nucléaire (ASN) et son expert I'Institut de radioprotection et de sûreté nucléaire (IRSN, en concertation avec les exploitants, mais également par l'ensemble des instances scientifiques et techniques internationales) ${ }^{5}$.

Il en a résulté une amélioration continue des installations, destinée à limiter l'étendue des accidents en renforçant la fiabilité des moyens mis en œuvre pour assurer le refroidissement des cœurs en toutes circonstances, en protégeant l'intégrité des enceintes de confinement (limitation de la pression en cas d'accident et de l'accumulation d'hydrogène par des procédés d'absorption robustes en toutes circonstances) et en réduisant les rejets en cas de dégonflage d'enceinte.

Ces évolutions techniques ont été accompagnées d'un effort considérable pour améliorer les actions automatiques de protection du cœur des réacteurs et donner aux opérateurs les informations utiles, en cas d'anomalies, pour comprendre l'évolution de l'installation. Des simulateurs de conduite en situations accidentelles sont utilisés systématiquement pour entraîner les équipes de conduite.

En ce qui concerne le parc français de réacteurs électronucléaires, les améliorations résultant des deux accidents de TMI et Tchernobyl, entre 1980 et 2000, ont été jugées adéquates au regard des défaillances constatées en 2011 à Fukushima, mais un durcissement supplémentaire des installations a été décidé afin de s'assurer d'une protection satisfaisante de l'environnement (pas d'impact durable) quelle que soit la gravité d'un accident concernant toutes les tranches d'un site, avec fusion des cœurs. Ce « noyau dur » (système additif de réfrigération du cœur indépendant et durci) et des aides à l'exploitation en situation dégradée (protection du personnel et arrivée rapide d'équipes de soutien formées et équipées) sont déjà partiellement mis en place et devraient être achevés avec l'ensemble des visites décennales du parc producteur d'électricité de l'exploitant public de la France.

\footnotetext{
${ }^{5}$ Voir dossier : Science of nuclear safety post-Fukushima, Guest editors Robert Dautray, Édouard Brézin. C. R. Physique, Académie des sciences, 13-4 (2012).
} 
En ce qui concerne l'accident de Mayak, mais aussi des accidents plus limités de criticité ou d'irradiation par des sources radioactives, le REX concerne particulièrement les contrôles de criticité, la composition des boves et effluents de retraitement (évolution des compositions chimiques, qualité de la réfrigération et du brassage), et l'inventaire et le suivi des matières radioactives et des sources.

Toute réflexion sur le démantèlement $d$ 'installations accidentées gravement en France doit intégrer ces évolutions, nombreuses, car elles sont destinées à limiter les conséquences d'un éventuel accident grave.

\section{Retours d'expérience des mises en sécurité et des démantèlements après accident}

Les démantèlements de réacteurs ou installations du cycle du combustible en fin d'exploitation sont déjà nombreux ${ }^{6}$ et les méthodes et équipements mis en œuvre sont déjà abondants. Mais l'accessibilité et les niveaux d'irradiation des réacteurs accidentés compliquent singulièrement les interventions et à ce jour aucun site accidentel n'a été totalement démantelé. II est utile de donner un bref rappel de leur historique, des mesures de mise en sécurité et des premières étapes du démantèlement dans chaque cas.

\subsection{Three Mile Island (réacteur à eau pressurisée de $900 \mathrm{MW}^{7}$}

\section{Séquence accidentelle}

Cet accident grave a commencé par un simple incident d'exploitation, une défaillance de l'alimentation en eau des générateurs de vapeur. Les automatismes de sûreté ont fonctionné parfaitement, mais deux défaillances successives ont conduit à l'accident grave : un oubli par un opérateur de commande d'ouverture d'une vanne, conduisant à une ouverture de la vanne de décharge du pressuriseur, suivie du coincement de celle-ci en position ouverte. L'analyse de cette succession d'événements a révélé que les opérateurs se sont appuyés sur de fausses informations, et qu'ils manquaient de renseignements sur l'état du cœur. Malgré une fusion partielle du cœur et d'importants relâchements de radioactivité dans l'enceinte de confinement, les conséquences radiologiques dans l'environnement ont été faibles. Elles n'ont pas conduit à l'évacuation des populations ${ }^{8}$.

\footnotetext{
${ }^{6}$ Voir l'annexe 1 du chapitre 1 du présent ouvrage.

${ }^{7}$ Accident de Three Mile Island : IRSN - Three Mile Island Unit 2, Recovery and clean up, by Chuck Negin, Washington Grove, Maryland, USA, charles negin@gmail.com; - http://www.irsn.

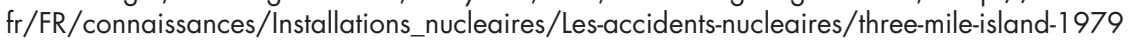

8 Évacuation des populations: Three Mile Island; the human dimension, edited by Vivian Shelanski.
} 
Après l'accident, le cœur était partiellement dégradé : les deux images (figure 9.2) présentent un schéma grossier de l'intérieur de la cuve de TMl avec la cavité dans le cœur et une photographie du fond de la cuve (au niveau du plénum supérieur) sur lequel sont plaquées les aiguilles combustibles brisées (11 mm de diamètre).
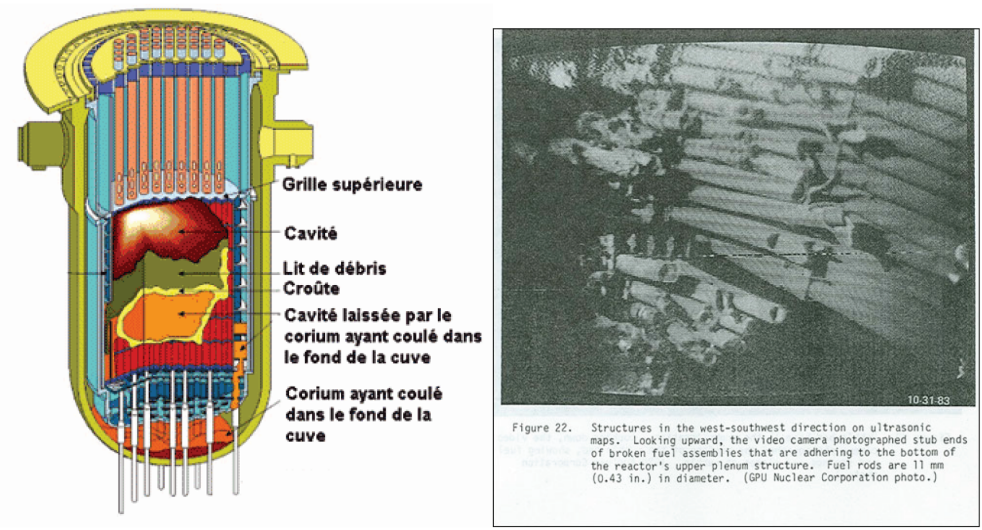

Figure 9.2

Le cœur de TMl - vue générale illustrant les dégâts et photo du fond de la cuve.

\section{Mise en sûreté de l'installation}

L'exploitant disposait d'une journée environ pour mettre en place une réfrigération pérenne du cœur, qui a commencé à se dégrader avec début de constitution d'un corium ${ }^{9}$, et pour éviter un perçage du fond de la cuve. Préoccupation supplémentaire, limiter la montée en pression du circuit primaire et la production d'hydrogène. Après cette mise en sûreté de l'installation, les travaux de démantèlement ont pu être étudiés, en prenant en compte la décroissance de puissance résiduelle ${ }^{10} \mathrm{du}$ cœur.

\section{Tableau 9.2}

Décroissance de la puissance résiduelle d'un réacteur de $1000 \mathrm{MW}$.

\begin{tabular}{|l|c|c|c|c|c|c|c|}
\hline Temps après l'arrêt & 1 heure & 1 jour & 1 mois & 1 an & 3 ans & 10 ans \\
\hline Puissance résiduelle $(\mathrm{kW})$ & 40000 & 16000 & 4000 & 280 & 50 & 28 \\
\hline
\end{tabular}

\footnotetext{
${ }^{9}$ Corium : mélange de combustible, de zirconium métallique ou oxydé et de matériaux des internes de la cuve.

10 Voir l'encadré 2 de : Réflexions sur l'avenir de l'énergie nucléaire, de la France d'aujourd'hui au monde de demain : Ile à IVe génération, par Robert Dautray, Jacques Friedel et Yves Bréchet. C. R. Physique 13-5 (2012), 480-518.
} 


\section{Premières étapes de démantèlement}

Il est difficile d'envisager une évacuation du corium avant un délai d'au moins 4 à 5 ans, en raison de la puissance résiduelle, d'autant plus que les intervenants bénéficient parallèlement $d$ 'une réduction par décroissance radioactive de l'inventaire radiologique, qui rendra les opérations de décontamination et l'accès plus faciles.

Dans le cas des PWR, contrairement aux BWR de Fukushima, la cuve est surmontée d'une piscine profonde qui permet une ouverture sous eau du couvercle de cuve.

À titre d'exemple, les opérations d'extraction du corium de TMI (1979) ont suivi le calendrier suivant:

- première inspection du bâtiment réacteur fin 1979 ;

- 1980 : relâchement contrôlé de l'atmosphère du bâtiment réacteur pour évacuer le krypton 85, un gaz rare de période 10,7 ans, et permettre un premier accès aux décontamineurs ;

- 1981 : épuration des eaux contaminées $\left(23000 \mathrm{~m}^{3}\right.$ pour extraire principalement le césium et le strontium) ;

- 1982 : première inspection de l'intérieur de la cuve sans l'ouvrir (vide de $9,5 \mathrm{~m}^{3}$ dans le cœur ;

- 1984 : ouverture de la cuve sous eau ;

- 1985 : exploration du fond de cuve (20 tonnes de débris et un corium solidifié) avec une activité de $35 \mathrm{mSv} / \mathrm{h}$ dans le hall ;

- de fin 1985 à 1986 : évacuations de 342 assemblages et des débris du cœur ;

- depuis 1991 : surveillance à long terme après épuration des eaux résiduelles et prise de " dispositions » vis-à-vis d'un résiduel du corium d'environ $1 \%$.

Ce démantèlement a été largement documenté et un inventaire établi pour les équipes de Fukushima ${ }^{1}$. De nombreux rapports détaillés peuvent être trouvés sur le site du gouvernement américain ${ }^{12}$. Cet accident, sans doute le plus représentatif pour nos installations, a eu trois caractéristiques particulières :

- c'était le premier de ce type, avec très peu de retours d'expérience d'accidents antérieurs, tous de niveau 4 ou 5 seulement;

\footnotetext{
${ }^{11}$ http://jolisfukyu.tokai-sc.jaea.go.jp/ird/sanko/file34.pdf

12 http://www.science.gov/topicpages/m/mile+island+cleanup.html
} 
- les technologies de téléopération étaient encore embryonnaires ;

- le volume contaminé (aérien ou aquatique) était bien au-delà des REX ;

- les déchets, hautement radioactifs, étaient de compositions souvent inconnues.

Ce bilan est très important, car il pose des questions ${ }^{13}$ auxquelles il serait nécessaire de répondre en cas d'éventuel accident grave similaire : maîtrise de la température du corium et des émissions d'hydrogène (avec son accumulation), détermination des contaminations résiduelles et éventuels rejets concertés pour permettre un accès aux opérateurs (niveau, information des populations, décision et suivi), épuration multi-nucléides des eaux contaminées, accès pour diagnostics avant ouverture de la cuve, moyens de décontamination (télé-opérés puis éventuellement manuels), diagnostics détaillés après ouverture de la cuve, moyens automatisés d'extraction des combustibles restants (intacts ou non) et du corium (cartographie du corium, caractéristiques physicochimiques, moyens de découpe, mise en conteneur), châteaux de transfert, mise en état sûr et surveillance de l'installation dans l'attente d'un démantèlement probablement beaucoup plus tard, après des études détaillées.

\subsection{Tchernobyl (réacteur graphite/eau de $1000 \mathrm{MW})^{14}$}

\section{Séquence accidentelle}

Cet accident a frappé en 1986 un réacteur de conception très différente de ceux construits en France ${ }^{15}$, avec un cœur UO2 faiblement enrichi dans une matrice graphite (faisant office de modérateur des neutrons) avec l'eau comme réfrigérant. La fusion du cœur et l'explosion, de nature chimique (explosion vapeur et hydrogène), ont résulté d'un emballement de la puissance (réactions en chaîne de l'uranium produisant de l'énergie de fission, à laquelle participe

\footnotetext{
${ }^{13}$ Ces questions ont été étudiées dans: 1 : Perrow C. (1999). Normal accidents, living with High risk technologies. Princeton University Press. Ces réflexions ont été reprises, dans un contexte plus général, dans : 2 : Perrow C. (2007-2011). The next catastrophe: reducing our vulnerabilities to natural, industrial, and terrorist disasters. Princeton University Press.

3: Posner R. A. (2004). Catastrophe, risk and response. Oxford University Press.

14 http://www.irsn.fr/FR/connaissances/Installations_nucleaires/Les-accidents-nucleaires/ accident-tchernobyl-1986/consequences-homme-environnement/Documents/irsn_tchernobyl_17-ans-apres.pdf

${ }^{15}$ Le concept de réacteur de Tchernobyl n'a été construit qu'en Union soviétique et dans les pays de l'Europe de l'Est alliés.
} 
le plutonium ${ }^{16}$ formé in situ par captures des neutrons sur l'isotope 238 de l'uranium) suite à un non-respect délibéré des règles de conduite.

Le principal REX de Tchernobyl concerne les plans d'urgence, la mise à l'abri des opérateurs et de la population sur de vastes espaces, l'évaluation des conséquences radiologiques, considérables compte tenu de l'excursion de puissance et du relâchement très rapide de la quasi-totalité des produits de fission (PF) volatils et gazeux mais également du rejet d'une portion significative des PF non volatils et des combustibles.

\section{Mise en sécurifé}

Les opérations de mise en sécurité ont consisté principalement en une évacuation de la population, puis, au niveau du réacteur, à un certain nombre $d$ 'actions d'urgence pour freiner les rejets radioactifs, confiner l'ensemble et redonner une certaine accessibilité autour de la zone du réacteur accidenté et de son site. Des décisions très difficiles ont été nécessaires avec des dépassements de doses considérables pour le personnel d'intervention dans les premières semaines, puis pour gérer une population très importante de "liquidateurs », 600000 en quatre ans, exposés à de fortes densités de radiations, avec une protection radiologique minime, gravement insuffisante. En sept mois, et dans l'urgence tragique, ceux-ci vont bâtir un «sarcophage » sur l'enceinte éventrée du réacteur, renfermant 190 tonnes environ de combustible nucléaire.
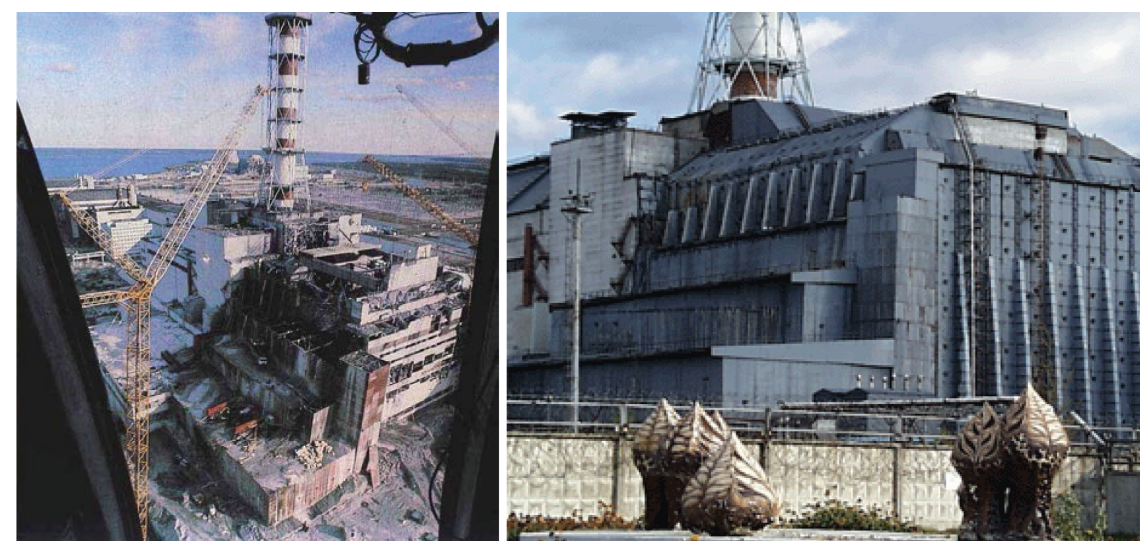

Figure 9.3

Vue du réacteur quelques semaines après l'accident et après construction du premier sarcophage.

${ }^{16}$ Cette précision est importante car ce plutonium sera dispersé par le considérable dégagement d'énergie de l'accident et déposé sur des surfaces substantielles, seul cas de tel phénomène dans un accident grave de réacteur à ce jour. 
La contamination des sols sera principalement gérée par grattage des zones les plus actives et empilement des terres correspondantes dans des zones réservées. Après assainissement du site de la centrale, les trois autres réacteurs ont été redémarrés et ont été exploités respectivement jusqu'à 1991,1996 et 2000.

Le corium solidifié est localisé au fond du bloc réacteur sous forme d'un magma de composition complexe (figure 9.4), mais, 29 ans après l'accident, aucune action $n^{\prime}$ a pu être encore entreprise pour le récupérer et démanteler l'ensemble du réacteur. Le risque de criticité suite à un effondrement du corium en présence d'eau est considéré comme très faible.

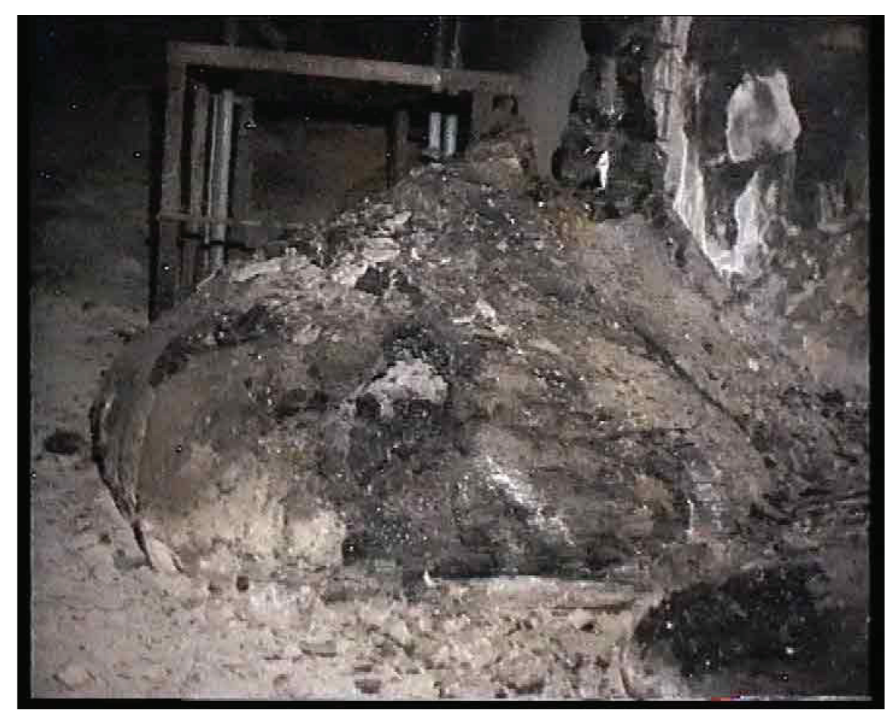

Figure 9.4

Le corium de Tchernobyl au fond du bloc réacteur.

Sur le site, sont encore entreposés les combustibles usés des trois autres réacteurs - qui pourraient être transférés vers un nouvel entreposage à sec sur site, encore à construire - et des quantités importantes de liquides radioactifs $\left(26000 \mathrm{~m}^{3}\right)$ et de déchets $\left(2400 \mathrm{~m}^{3}\right)$ de compositions mal connues.

\section{Préparation du démantèlement}

Le site est sous la menace d'un effondrement de l'enceinte de confinement qui pourrait provoquer la dispersion dans l'environnement d'une grande quantité de poussières radioactives incluant du combustible (5 à 10 tonnes?). La politique scientifique et technique adoptée consiste aujourd'hui, près de 
30 ans après I'accident, à préparer un démantèlement sous une nouvelle enceinte de confinement, en cours de construction (figure 9.5), qui recevra l'équipement télé-opéré permettant de démanteler l'enceinte actuelle et le bâtiment réacteur ruiné, puis $d^{\prime}$ accéder au corium avec un minimum d'intervention humaine. Étudiée depuis 2008, sa construction a débuté en avril 2012 avec pour maître d'œuvre du projet la coentreprise Novarka, détenue à parts égales par les sociétés françaises Vinci Construction Grands projets et Bouygues Travaux publics. Compte tenu des difficultés de financement, elle ne devrait être achevée qu'en 2017.
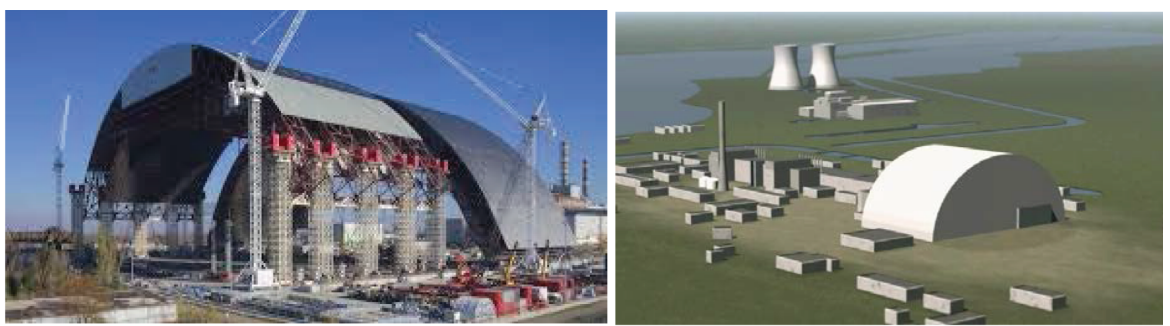

\section{Figure 9.5}

La nouvelle arche en construction (2014) et après mise en place prévue (2017).

La nouvelle enceinte en forme d'arche est montée en deux phases sur un terrain contigu de la centrale, après décontamination soignée du sol. Posée sur deux longrines en béton, les segments de l'arche, une fois assemblés, seront glissés au-dessus du sarcophage existant. Ses dimensions $1118 \mathrm{~m}$ de haut, $152 \mathrm{~m}$ de long et une portée de $257 \mathrm{~m}$ ) et son poids, 25000 tonnes, sont hors du commun.

Les objectifs principaux de cette enceinte sont notamment le confinement des matières radioactives, la protection des travailleurs sur site et la protection du sarcophage existant contre les agressions climatiques. Elle abritera les équipements et installations qui permettront le démantèlement en limitant le plus possible les interventions humaines. Aucune information précise sur ce démantèlement, son calendrier, son coût n'est disponible aujourd'hui, et la persistance des difficultés du secteur énergétique ukrainien, l'ampleur de la tâche à accomplir et son coût laissent penser que le démantèlement sera très long, malgré l'importance des coopérations internationales. 


\subsection{Fukushima (quatre réacteurs à eau bouillante de 400 à $550 \mathrm{MW})^{17}$}

\section{Séquence accidentelle}

L'accident correspond à une configuration très différente, puisque concernant un site dans son ensemble suite à un évènement exceptionnel, un séisme majeur, auquel les réacteurs ont correctement réagi (avec arrêt automatique) et résisté. Mais le séisme de mars 2011 a provoqué un tsunami d'ampleur considérable, à l'origine de l'accident qui a dévasté simultanément quatre des six réacteurs du site. Ce risque avait été identifié mais très insuffisamment pris en compte par l'opérateur de la centrale et l'Autorité de sûreté japonaise. L'accident a eu des conséquences environnementales importantes au dénoyage et à la fusion quasi complète de quatre des six cœurs (les deux réacteurs les plus récents n'ayant pas été accidentés), à la montée en pression des enceintes de confinement au-delà des valeurs de dimensionnement, au dégagement de grandes quantités d'hydrogène conduisant à des réactions chimiques exothermiques, puis à des déflagrations conduisant à des explosions chimiques, avec de plus des risques de dénoyage des piscines de stockage des combustibles usés.

\section{Mise en sûreté de l'installation}

Une fois la réfrigération des cœurs assurée (et celle des éléments combustibles usés entreposés dans les piscines), le Japon a donné priorité en 2011 à la stabilisation de la situation sur le site et à sa décontamination pour donner accès aux zones dévastées puis mettre définitivement les installations en situation d'arrêt sûr en fin 2011. Les travaux réalisés et en cours sont considérables et des étapes importantes de leur programme ont été achevées dans les délais ${ }^{18}$, comme l'évacuation des débris générés par les explosions, la vidange de la piscine de stockage des combustibles irradiés du réacteur 4 (voir figure 9.6) avec construction d'un hall confiné de manutention en surplomb de la piscine.

$17 \mathrm{hHp}: / /$ www.irsn.fr/FR/connaissances/Installations_nucleaires/Les-accidents-nucleaires/ accident-fukushima-2011/fukushima-2014/Documents/irsn_fukushima_point_situation_201403.pdf et Académie des sciences (2012). L'accident majeur de Fukushima, groupe de travail présidé par Alain Carpentier avec Étienne-Émile Baulieu, Édouard Brézin et Jacques Friedel. EDP Sciences.

${ }^{18}$ Guidez J. et al. (2014). Le démantèlement de la centrale de Fukushima : un long processus. Revue générale nucléaire $\mathrm{n}^{\circ} 5$. 


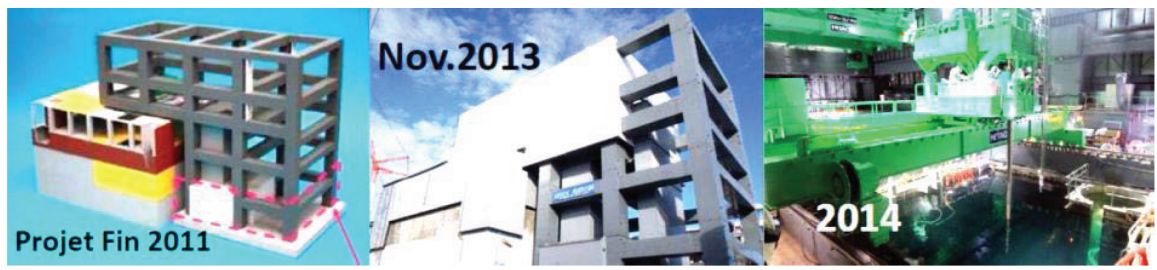

\section{Figure 9.6}

Construction d'un hall de manutention en surplomb de la piscine de stockage des combustibles usés du réacteur $n^{\circ} 4$ de Fukushima.

Des méthodes très innovantes sont utilisées, comme la radiographie muon du réacteur 1 pour localiser le corium, la réalisation autour des quatre tranches, d'une muraille souterraine de terre gelée descendant jusqu'à une couche géologique étanche à $27 \mathrm{~m}$ de profondeur, destinée à confiner et à récupérer les fuites et la mise en service (difficile) d'une épuration multi-nucléides des effluents liquides.

Une difficulté importante du point de vue du REX résulte de l'accumulation d'un volume considérable d'effluents liquides et de déchets, sans que des exutoires aient encore été identifiés : la rigidité réglementaire qui s'impose dans ce domaine est un frein réel à la préparation d'un démantèlement.

\section{Premières étapes du démantèlement}

Dès fin 2011 , un calendrier prospectif du démantèlement a été proposé par l'opérateur, Tepco (figure 9.7), définissant les principales étapes et leur contenu. Ce calendrier est aujourd'hui respecté et s'étend sur une quarantaine d'années. Il mobilise environ 6000 à 8000 personnes sur le site.

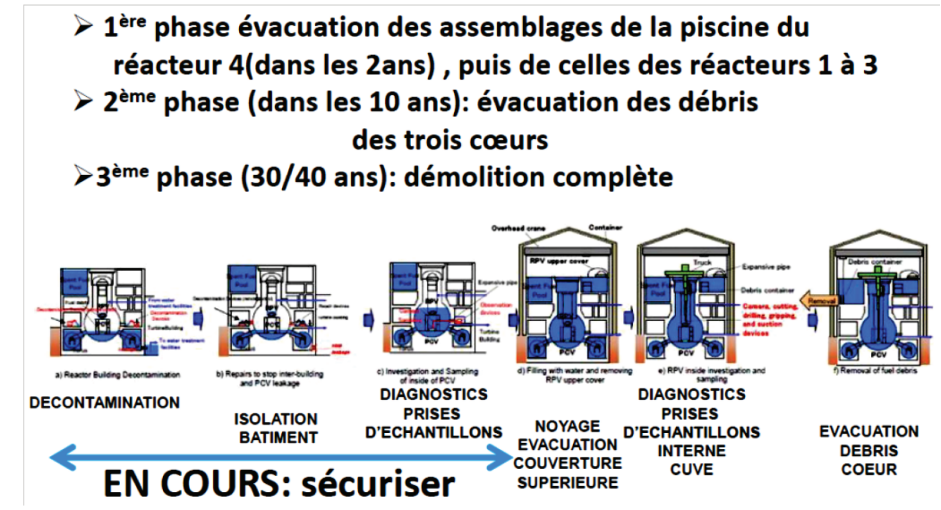

Figure 9.7

Calendrier prospectif du démantèlement de Fukushima. 
Pour effectuer les recherches et développements nécessaires à la préparation des démantèlements, un institut dédié a été créé, l'International Research Institute for nuclear Decommissionning (IRID ${ }^{19}$ ), qui s'appuie sur deux panels d'experts internationaux (management et expertise technique) et a initié en 2013 un concours d'idées pour l'élimination des déchets des cœurs. C'est une initiative qui méritera un suivi attentif.

La priorité a été donnée à trois chantiers :

- l'évacuation des combustibles des piscines de stockage des quatre réacteurs, fragilisées par les explosions (objectif 2017/2018 pour les quatre réacteurs);

- la préparation de l'extraction des débris des coriums ;

- le traitement et le stockage des déchets radioactifs liquides et solides.

Pour chacune de ces priorités, un éventail large de méthodes et équipements à développer a été identifié et les études lancées en s'appuyant systématiquement sur des entreprises, nationales ou non, capables de les réaliser. II est fait largement appel à des engins télé-opérés et, compte-tenu des délais ambitieux de récupération des coriums, à des engins très durcis aux rayonnements.

\section{|Conclusion et perspectives}

Chaque accident reste un cas particulier, et les premières mesures sont toujours la mise en sécurité des populations et la mise en sécurité de l'installation elle-même. Et ce, avant toute opération de démantèlement. Pour se mettre en perspective, on ne peut ignorer les enseignements souvent très complémentaires acquis sur les trois accidents ci-dessus, qui ont été analysés en grand détail par les Autorités de sûreté nucléaire et leurs experts, en concertation avec les exploitants. II en est résulté une amélioration continue des installations, destinée à limiter l'étendue des accidents en renforçant la fiabilité des moyens mis en œuvre pour assurer le refroidissement des cœurs en toutes circonstances, en protégeant l'intégrité des enceintes de confinement (limitation de la pression en cas d'accident et de l'accumulation d'hydrogène) et en réduisant les rejets en cas de dégonflage d'enceinte.

$C^{\prime}$ est ainsi qu'en France, un durcissement supplémentaire des installations a été décidé afin de s'assurer d'une protection satisfaisante de l'environnement (pas d'impact durable) quelle que soit la gravité d'un accident concernant toutes les tranches d'un site, avec fusion des cœurs. Ce «noyau dur » repose sur un système additif de réfrigération du cœur, indépendant et durci,

${ }^{19}$ Voir: http://irid.or.jp 
et sur des aides à l'exploitation en situation dégradée (protection du personnel et arrivée rapide d'équipes de soutien formées et équipées).

Dans le cas où le cœur accidenté est resté confiné dans la cuve, le retour d'expérience de TMI permet d'identifier les principaux « challenges » et installations ou équipements nécessaires à sa prise en charge. Les opérations de nettoyage et démantèlement peuvent s'étaler sur des dizaines $d^{\prime}$ 'années mais une réflexion amont, avec des essais de qualification sur installations intactes disposant d'une bonne accessibilité, pourrait permettre une meilleure approche prédictive des interventions suite à un accident.

- La maîtrise de la température du corium et des émissions d'hydrogène a été facilitée par le fait que les sources électriques étaient toujours disponibles et ont pu être utilisées dès le diagnostic établi : I'urgence de rétablir l'alimentation électrique et la réfrigération du cœur est primordiale.

- Le risque hydrogène était mal évalué : la destruction de cet hydrogène par recombinaison doit accompagner l'action ci-dessus.

- Des rejets concertés peuvent être nécessaires en cas de montée en pression excessive du hall. Ces rejets doivent être aussi tardifs que possible (réduction des rejets d'iode en particulier) et concertés (limitation des doses dans l'environnement). La mise en place de filtres adaptés à l'atmosphère post-accidentelle de l'enceinte de confinement, en particulier pour les iodes et le césium, permet d'améliorer très substantiellement la situation.

- Une décontamination du hall par des engins robotisés, au moins au début, puis avec intervention humaine et fixation de la contamination résiduelle pour protéger les intervenants du risque d'inhalation, facilite l'accès pour diagnostic de la situation avec des dosimétries modérées.

- Les eaux de fuites, fortement radioactives, peuvent avoir envahi les soussols des réacteurs, voire le bâtiment énergie en cas de rupture des tubes des générateurs de vapeur : leur décontamination peut requérir une multiplicité de systèmes additifs. Par exemple à TMI :

- une installation immergée pour les eaux les plus radioactives de la piscine (zéolite) ;

- une filtration des particules fines radioactives en suspension dans l'eau primaire lors de l'extraction du corium (zéolite et charbon actifs) ;

- un traitement de l'eau primaire contre une contamination biologique afin de maintenir une eau claire, en particulier lors de l'extraction du corium ;

- les résines échangeuses d'ions de l'installation pour les eaux faiblement contaminées. 
Ces systèmes, essentiels, doivent être dimensionnés pour des quantités très importantes d'effluents, deviennent très radioactifs eux-mêmes, doivent être largement télé-opérés, et sont adossés à tout un ensemble de procédures et équipements pour récupérer les filtres, les bloquer dans des conteneurs agréés pour des sites $d^{\prime}$ entreposages dont les critères $d^{\prime}$ acceptation sont souvent très contraints.

Les normes de rejets actuelles, très restrictives, demandent des filtrations multi-nucléides : leur mise en service à Fukushima a été longue et difficile.

- Les eaux filtrées et traitées, peu radioactives mais tritiées, représentent des volumes considérables et leurs rejets présentent des difficultés sociales importantes: TMl a adopté une installation d'évaporation avec un rejet contrôlé. La politique de Fukushima, qui stocke plus de $400000 \mathrm{~m}^{3}$ d'effluents liquides, n'apparaît pas encore clairement, la protection du milieu halieutique étant primordiale dans le pays.

- L'accès au corium se fait au bout de quelques années (entre 3 et 4 ans à TMI) en deux étapes, après décontamination extensive du hall du réacteur :

- accès limité par des traversées existantes pour imagerie, prélèvements et cartographie ;

- ouverture du couvercle pour extraction du corium. L'accès au fond de cuve impose des outils de grande longueur : le choix à TMl a été de mettre un plancher à quelques mètres du sommet de la cuve.

- Le corium peut être très dur et mêlé aux débris des structures tels que les grilles: la variété des textures et compositions exige une souplesse $\mathrm{d}^{\prime}$ approche (figure 9.8). TMI a opté pour des technologies de forage standard mais des méthodes plus actuelles peuvent être envisagées (suivre le chantier Fukushima). Les quantités à remonter sont importantes, supérieure à 100 tonnes, ce qui requiert un grand nombre de mouvements des châteaux de transfert et de conteneurs blindés d'entreposage, et un chantier de plusieurs années (TMI : plus de 5 ans).

- L'extraction du corium n'est cependant que partielle, car des débris de combustibles peuvent se trouver en de nombreux points du circuit primaire (GV, pressuriseurs, tuyauteries), très difficiles d'accès. À TMI, I'estimation porte sur environ une tonne de débris résiduels, qui ne seront pris en charge que lors du démantèlement complet de l'installation. Ils doivent être cartographiés en faisant appel à un large panel de détecteurs (gamma, neutrons actifs et passifs, prélèvements et mesures alpha) et à de la vidéo. 


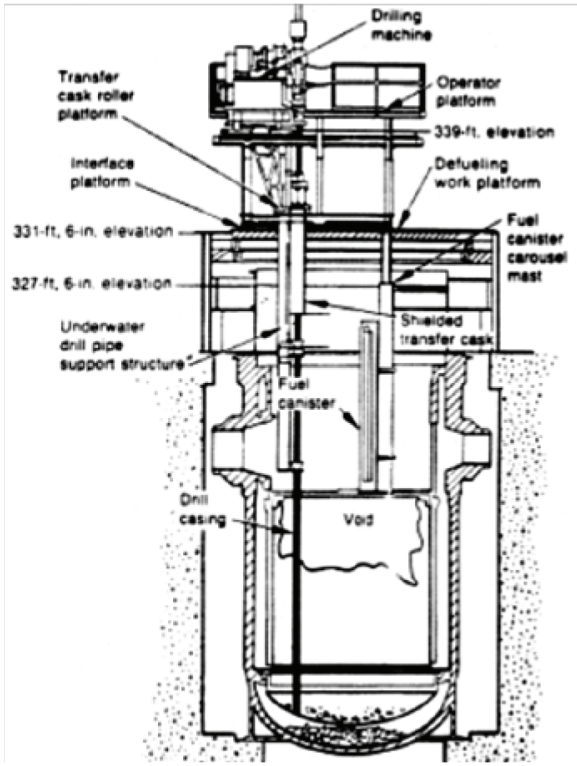

Figure 9.8

TMl : Plateforme de forage au-dessus de la cuve.

- La maîtrise de la criticité et du risque hydrogène sont, avec la dosimétrie du personnel, les contraintes de sûreté principales.

- Le démantèlement complet de l'installation doit faire la part entre une volonté de nettoyer définitivement l'installation et celle de réduire les doses pour le personnel. À TMI, il est envisagé environ 55 ans après l'accident (décroissance du Co60 en particulier).

- De nombreuses filières de déchets doivent être activées avec le support du gouvernement, car certains ne respectent pas les critères réglementaires.

L'accident de Fukushima a de plus mis en évidence l'intérêt d'une analyse approfondie de la résistance des piscines d'entreposage des combustibles usés adjointes à chaque tranche, compte tenu de l'importance de leur inventaire radiologique en produits de fission de vie longue, et d'une évacuation aussi rapide que possible de ces combustibles dans l'hypothèse d'une fragilisation des bâtiments et moyens de réfrigération, ou d'une difficulté d'accès pour maintenance.

Lorsque le cœur n'est pas resté confiné dans la cuve, il serait nécessaire de pouvoir explorer les locaux sous-jacents, d'évaluer la quantité de corium perdu et sa position, de pouvoir faire des prélèvements pour le caractériser car il aura été au contact du béton. La récupération de ce corium ne pourrait 
sans doute être engagée qu'après évacuation de la cuve, l'accès au puits de cuve par des engins automatisés étant probablement très complexe. Le retour d'expérience de Fukushima, rendu encore plus compliqué en raison de la complexité du bloc cœur et de l'enceinte de confinement de cette génération de BWR, sera essentiel.

Par rapport à TMI, une complexité supplémentaire, constatée à Tchernobyl et Fukushima, résulte du niveau d'irradiation, très supérieur, et de la contamination extérieure : les moyens de diagnostic et $d^{\prime}$ intervention doivent être durcis au rayonnement, et les moyens de décontamination doivent être développés, en particulier pour les sols.

En conclusion et de manière non exhaustive, on peut s'interroger sur les problématiques qui apparaissent dans ce type de chantier, afin d'améliorer l'efficacité des interventions en situations accidentelles:

- quelles dosimétries seraient acceptables pour le personnel en situation d'urgence et dans quelles conditions (niveau maximal, volontariat, protections spécifiques, assistance lors des interventions) ?

- comment accélérer les procédures d'autorisation des chantiers afin de réduire les conséquences radiologiques en cas d'accident lexemple du remarquable chantier d'évacuation des combustibles du réacteur à Fukushima)?

- les règles concernant les rejets devraient être assouplies dans ces circonstances pour permettre aux intervenants un accès plus rapide au hall du réacteur pour diagnostics et actions d'urgence. On peut noter que, pour réduire la durée des chantiers et réduire ainsi les dosimétries collectives, les Japonais ont décidé de ne pas recouvrir d'une nouvelle enceinte les réacteurs 2 et 3 pour le chantier de déblayage des débris et la préparation à l'accès aux piscines de stockage des combustibles. Ils envisageraient de faire de même sur le réacteur 1 en démontant l'enceinte provisoire actuelle ;

- les normes radiologiques pour la population se révèlent excessivement contraignantes, sans bénéfice sanitaire évident, les évacuations provocant un stress important. La différence entre $1 \mathrm{mSv}$, niveau d'excellence imposé aux exploitants, et 20 ou 50 mSv, niveaux quasiment sans risques, devrait être mieux expliquée et comprise afin d'éviter des évacuations excessives et des mouvements de panique ;

- le stockage des terres, matériaux divers et végétaux contaminés par le césium, est relativement simple d'un point de vue technologique car il s'agit de contaminations limitées et d'un contaminant principal, le 
césium, qui se fixe assez aisément. Mais il exige une capacité de décision forte des pouvoirs publics pour autoriser des stockages temporaires ou définitifs : prévoir a priori des espaces dédiés contigus aux centrales et susceptibles de recevoir en urgence et de manière temporaire des déchets (avec protection du sous-sol)?

- la question du rejet des effluents liquides épurés est critique à Fukushima en raison d'interdictions excessives de rejets en mer, alors que la lixiviation des sols contaminés est déjà réelle : cette question devrait être réglée au niveau international, les règles actuelles étant inadaptées à de telles situations et les populations inutilement inquiétées par des interdictions de rejet léonines.

Le retour d'expérience des opérations en cours à Fukushima, et de l'étude épidémiologique menée en parallèle de toutes ces opérations, sera extrêmement précieux pour répondre aux questions qui restent posées par ce type de chantier. 